\title{
3D Photonic Nanostructures via Diffusion-Assisted Direct fs Laser Writing
}

\section{Gabija Bickauskaite, ${ }^{1,2}$ Maria Manousidaki, ${ }^{2,3}$ Konstantina Terzaki,, 4 Elmina Kambouraki, ${ }^{2,4}$ Ioanna Sakellari, ${ }^{2,3}$ Nikos Vasilantonakis, ${ }^{2,4}$ David Gray, ${ }^{2}$ Costas M. Soukoulis, ${ }^{2,5}$ Costas Fotakis, ${ }^{2,3}$ Maria Vamvakaki, ${ }^{2,4}$ Maria Kafesaki, ${ }^{2,4}$ Maria Farsari, ${ }^{2}$ Alexander Pikulin, ${ }^{6}$ and Nikita Bityurin ${ }^{6}$}

\author{
${ }^{1}$ Department of Quantum Electronics, Vilnius University, 02300 Vilnius, Lithuania \\ ${ }^{2}$ IESL-FORTH, N. Plastira 100, Heraklion, 70013 Crete, Greece \\ ${ }^{3}$ Department of Physics, University of Crete, Heraklion, 71003 Crete, Greece \\ ${ }^{4}$ Department of Materials Science and Technology, University of Crete, Heraklion, 71003 Crete, Greece \\ ${ }^{5}$ Ames Laboratory, Department of Physics and Astronomy, Iowa State University, Ames, IA 50011-2011, USA \\ ${ }^{6}$ Institute of Applied Physics, Russian Academy of Sciences, Nizhny Novgorod, Russia
}

Correspondence should be addressed to Maria Farsari, mfarsari@iesl.forth.gr

Received 28 May 2012; Revised 24 July 2012; Accepted 24 July 2012

Academic Editor: Natalia M. Litchinitser

Copyright ( 2012 Gabija Bickauskaite et al. This is an open access article distributed under the Creative Commons Attribution License, which permits unrestricted use, distribution, and reproduction in any medium, provided the original work is properly cited.

\begin{abstract}
We present our research into the fabrication of fully three-dimensional metallic nanostructures using diffusion-assisted direct laser writing, a technique which employs quencher diffusion to fabricate structures with resolution beyond the diffraction limit. We have made dielectric 3D nanostructures by multiphoton polymerization using a metal-binding organic-inorganic hybrid material, and we covered them with silver using selective electroless plating. We have used this method to make spirals and woodpiles with $600 \mathrm{~nm}$ intralayer periodicity. The resulting photonic nanostructures have a smooth metallic surface and exhibit well-defined diffraction spectra, indicating good fabrication quality and internal periodicity. In addition, we have made dielectric woodpile structures decorated with gold nanoparticles. Our results show that diffusion-assisted direct laser writing and selective electroless plating can be combined to form a viable route for the fabrication of 3D dielectric and metallic photonic nanostructures.
\end{abstract}

\section{Introduction}

Direct fs laser writing is a technique that allows the construction of three-dimensional micro-and nanostructures [1]. It is based on the phenomenon of multiphoton absorption and subsequent polymerization; the beam of an ultrafast laser is tightly focused into the volume of a photosensitive material, initiating multiphoton polymerization within the focused beam voxel. By moving the beam three-dimensionally, arbitrary 3D, high-resolution structures can be written. By simply immersing the sample in an appropriate solvent, the unscanned, unpolymerized area can be removed, allowing the $3 \mathrm{D}$ structure to reveal. A variety of applications have been proposed including microfluidics [2], micro-optics [3, 4], scaffolds for biomolecules and cells [5-7], and photonics and metamaterials [8-10].

There has been a lot of research efforts to improve the resolution of DLW technology, which for a long time has been in the range of $100 \mathrm{~nm}$. The method which most successfully and substantially has increased the resolution not only of single lines but also of 3D structures is DLW inspired by stimulated-emission-depletion (STED) fluorescence microscopy $[11,12]$. In STED-DLW, two laser beams are used; one is used to generate the radicals, and the second beam to deactivate them. Several schemes have been proposed including single-photon (rather than multiphoton) excitation [13], a one-color scheme [14] and multiphoton two-color scheme $[15,16]$. Structures with 


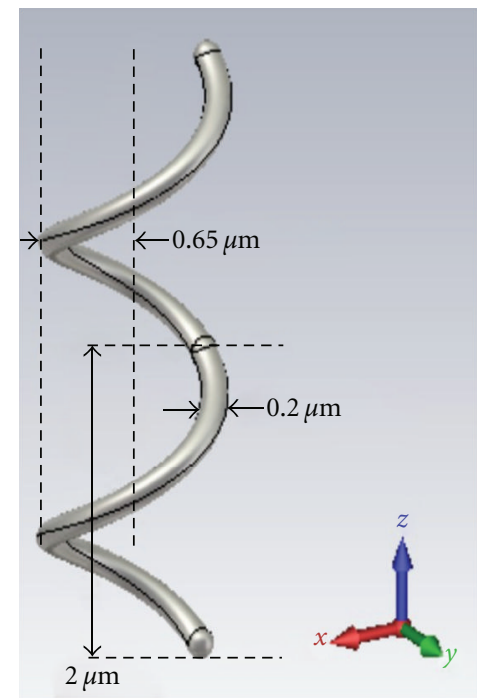

FIgure 1: The design of the 3D spirals.

very high resolution and very small intralayer distances have been fabricated using this approach. However, the implementation of DLW-STED is complicated, requiring very fine beam control and specialized photoinitiators which not only have high two-photon cross-section, but also high fluorescence quantum efficiency $[15,17]$. Consequently, only geometrically simple structures have been fabricated to-date.

Our team has shown recently that it is possible to increase the writing resolution of multiphoton polymerization by employing diffusion-assisted DLW (DA-DLW), a scheme based on quencher diffusion, in a chemical equivalent of STED [18]. This is based on the combination of a mobile quenching molecule with a slow laser scanning speed, allowing the diffusion of the quencher in the scanned area, the depletion of the generated radicals, and the regeneration of the consumed quencher. The material used as quencher is 2-(dimethylamino) ethyl methacrylate (DMAEMA), an organic monomer which is also part of the polymer structure. Due to its amine moieties, this is the same monomer we have employed in the past as a metal ligand, to enable the selective metallization of 3D photonic crystals [19]. In general, metallic nanostructures are very interesting due to their potential electromagnetic functionalities, which are not observed in bulk materials [20-23]. Metallic periodic nanostructures can significantly modify the properties of light with wavelength close to their periodicity, resulting in potential applications in scientific and technical areas such as filters, optical switches, sensing, imaging, energy harvesting and photovoltaics, cavities, and efficient laser design [24]. Several fabrication techniques have been employed for the fabrication of such structures, including colloidal lithography, [25] focused ion beam drilling [26], photopolymerization and photoreduction [27], and others [28]. Our approach was to fabricate $3 \mathrm{D}$ dielectric nanostructures containing the metal binding material DMAEMA and subsequently selectively metallize them with silver using electroless plating (EP). EP is a fairly simple process that does not require any specialized equipment, and the metal deposition can be done without using any electrical potential $[29,30]$. In general, it is characterized by the selective reduction of metal ions at the surface of a catalytic substrate immersed into an aqueous solution of metal ions, with continued deposition on the substrate through the catalytic action of the deposit itself. Using DLW and selective EP, we successfully fabricated 3D metallic photonic crystals with bandgaps at optical wavelengths [31].

In this paper, we combine these two methodologies to fabricate 3D metallic and structures with complex geometries and subdiffraction limit resolution. We fabricate woodpile and spiral photonic crystals, and we show that they have well-defined diffraction patterns, indicating the quality of their fabrication and their internal periodicity. In addition, we have fabricated 3D structures decorated with gold nanoparticles. Such structures can be useful in applications such as biosensing.

\section{Design}

In this paper, we present three kinds of nanostructures.

(i) Silver-coated woodpile structures with a period of $600 \mathrm{~nm}$ : these type of structures were investigated theoretically and experimentally in [31], and they were found to have bandgaps at optical wavelengths.

(ii) Dielectric woodpile structures, also with period $600 \mathrm{~nm}$, decorated with gold nanoparticles: these can be useful in applications such as biosensing, where thiol chemistry can be employed for biomolecule immobilization [32].

(iii) Spiral photonic structures: these were modeled on the structures presented in [33] by Ganzel and colleagues from KIT, Germany (Figure 1). In the KIT study, voids were fabricated into a positive photoresist using DLW, which were subsequently filled with gold using electroplating. Their structures were used as broadband polarizers. In our study, we have copied the spiral design and used a metal-binding negative photopolymer to recreate these spiral structures. As these spirals have high aspect ratio and it is difficult for them to remain free standing during the sample development process, support structures were added to the design, as it will be shown in the Results section.

\section{Fabrication}

The materials investigation, synthesis, and metallization protocols employed have been described in detail previously in $[18,19,31]$. The silver-coated structures were fabricated using 30\% DMAEMA [19], while the gold-nanoparticlecovered ones 10\% DMAEMA [18]. The gold nanoparticles were prepared following the metallization process described in [19], omitting the last plating step.

For the fabrication of the 3D nanostructures, a Ti: Sapphire femtosecond laser $(800 \mathrm{~nm}, 75 \mathrm{MHz},<20 \mathrm{fs})$ was 


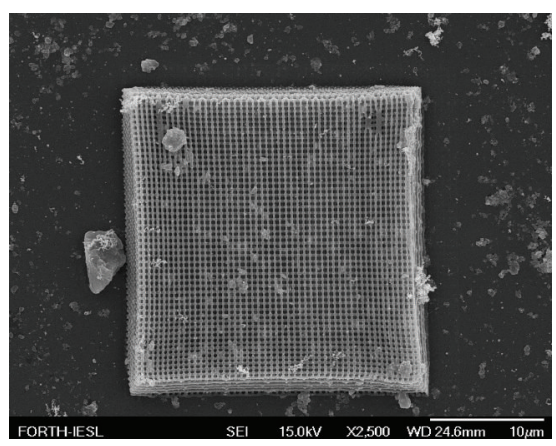

(a)

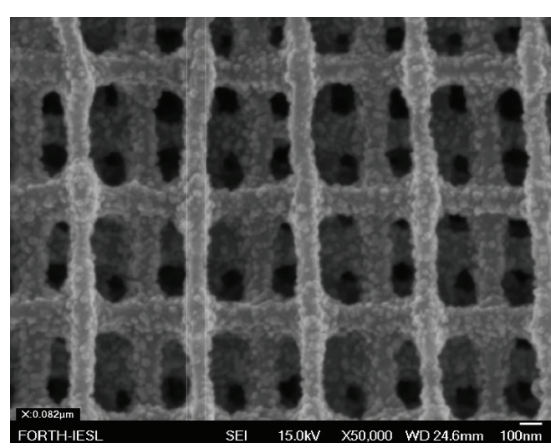

(b)

FIgure 2: Woodpile photonic crystals: (a) the whole structure, (b) detail.

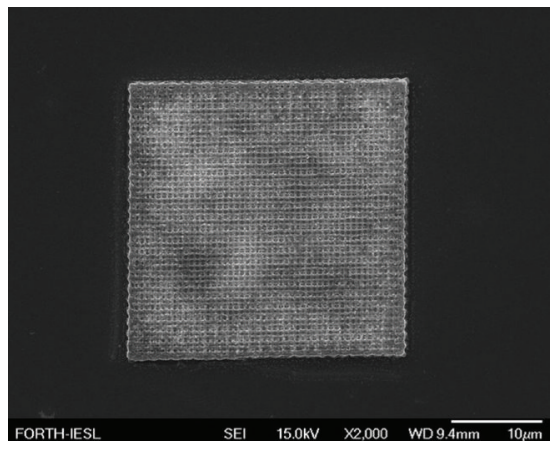

(a)

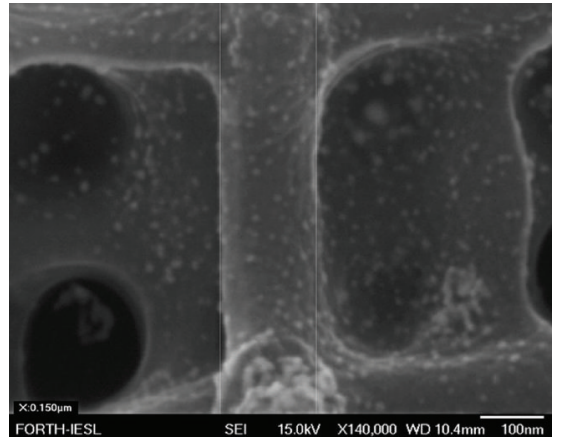

(b)

FIGURE 3: Woodpiles decorated with gold nanoparticles (a) the whole structure, (b) detail.

focused into the photopolymerisable composite using a high numerical aperture focusing microscope objective lens $(100 \mathrm{x}$, N.A. = 1.4, Zeiss, Plan Apochromat). Sample movement was achieved using piezoelectric and linear stages, for accurate and step movement, respectively (PI). The whole DLW setup, which is described in detail in [34], was computer controlled using the 3DPoli software. Here, the average laser power used for the fabrication of the high-resolution woodpile structures was $1.85 \mathrm{~mW}$, measured before the objective, while the average transmission to the sample was $20 \%$. For the spiral structures, the average power was increased to $5.5 \mathrm{~mW}$, scanning the beam at $10 \mu / \mathrm{s}$ and $20 \mu / \mathrm{s}$ to write the spirals and the supports, respectively. To avoid contact with the lens immersion oil, all structures were fabricated upside down with the glass substrate in contact with the oil. They were built in a layer-by-layer fashion starting from the top with the last layer adhering to the substrate. This way, the laser beam did not cross an already polymerized layer, causing second polymerization or beam distortion.

\section{Diffraction Spectra}

To check the quality of the structures, we used the diffraction pattern in the transmitted waves produced by the structure when illuminated using a white light beam. In general, diffraction patterns reveal structural characteristics as well as sample quality [35]. For this, a home-built setup was employed, built according to [36, 37]. Light from a Ti:Sapphire laser $(800 \mathrm{~nm}, 180 \mathrm{fs}, 1 \mathrm{~mJ} /$ pulse, $1 \mathrm{KHz}$ repetition rate) was focused using an $f=3 \mathrm{~cm}$ lens into a $3 \mathrm{~cm}$ long cell filled with distilled water, in order to produce white light continuum, providing a useful broad spectral range of $450 \mathrm{~nm}$ to $1000 \mathrm{~nm}$ wavelength. The light was collimated and then focused on the sample. The sample was mounted to have accurate $3 \mathrm{D}$ and rotational control. The half-opening angle of the incident light was reduced to $5^{\circ}$, assured by iris diaphragms.

\section{Results and Discussion}

Figure 2(a) shows a scanning electron microscopy (SEM) image of a woodpile structure with $600 \mathrm{~nm}$ period fabricated and metallized using the procedure described earlier. Figure 2(b) shows a detail of such a structure. It can be seen that the resolution achieved is in the order of $100 \mathrm{~nm}$.

Figure 3 shows SEM images of woodpile structures decorated with gold nanoparticles. Figure 3(a) shows the whole structure, while Figure 3(b) shows a detail of such a structure, where the nanoparticles are clearly visible. The density of these nanoparticles can increase or decrease by increasing or decreasing the percentage of DMAEMA, respectively [19]. The size of the nanoparticles can be modified by altering the growing conditions [19]. 


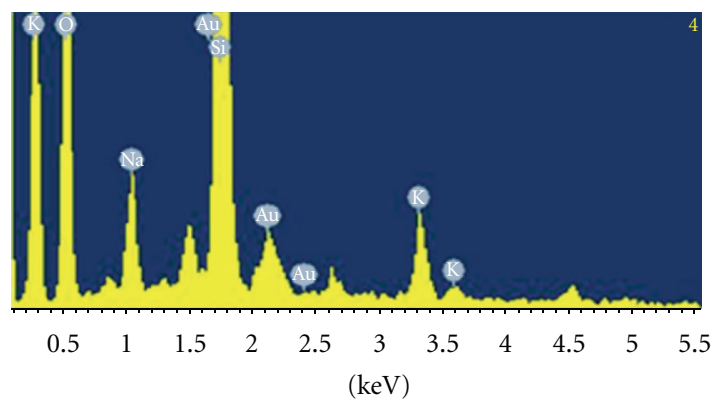

Full-scale 409 cts cursor: 0.000

FIGURE 4: EDX spectrogram of the woodpile structures. The gold ( $\mathrm{Au})$ peaks are clearly visible.

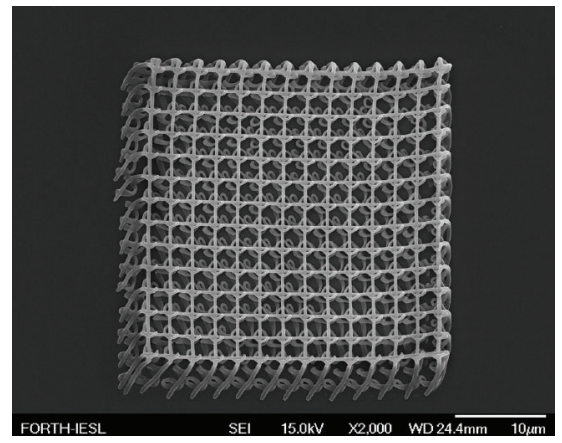

(a)

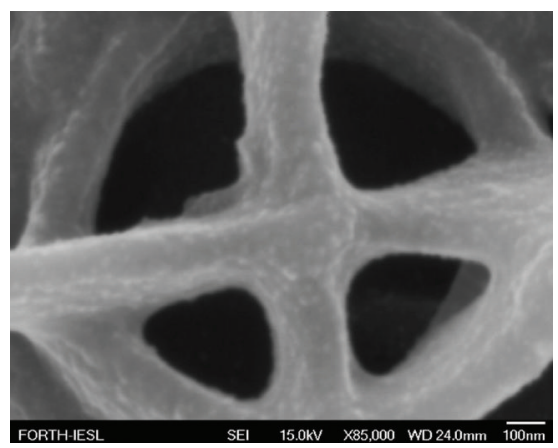

(c)

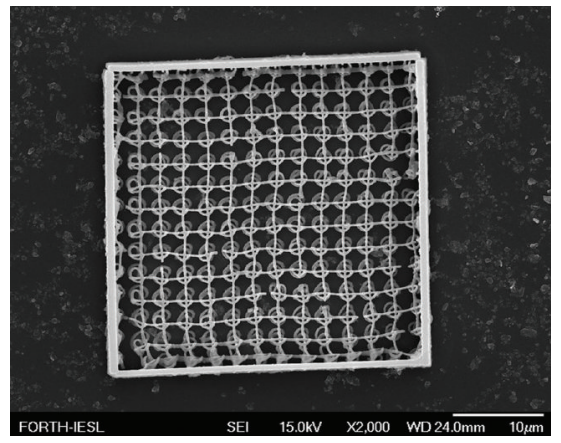

(b)

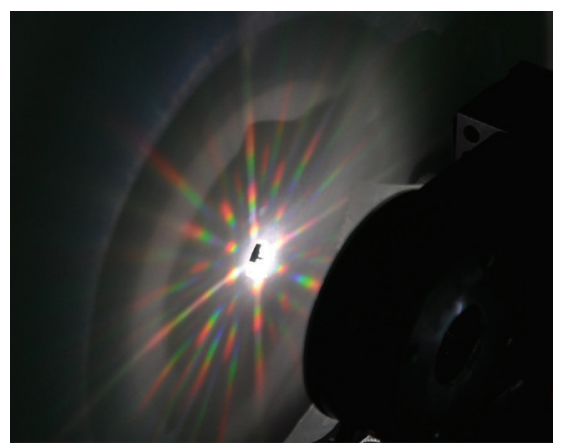

(d)

Figure 5: Spiral photonic crystal structures. (a) An array of dielectric spirals. (b) An array of metalized spirals. (c) Detail of a spiral with lateral resolution is in the order of $100 \mathrm{~nm}$. (d) The diffraction pattern generated when the structure is illuminated with white light.

Figure 4 shows the energy-dispersive X-ray (EDX) spectrogram of the woodpile structures, where the gold $(\mathrm{Au})$ peaks are clearly visible.

Figure 5 shows a series of dielectric and silver-coated spiral structures fabricated as described earlier. Figures 3(a) and $3(\mathrm{~b})$ show $14 \times 14$ arrays of spirals before silver coating, respectively. Support lines between the spirals are clearly visible. It is also clear that even thought there is some debris on the glass substrate, the silver metallization is selective. Figure 3(c) shows a close SEM image of a spiral; the resolution achieved is in the order of $100 \mathrm{~nm}$. In addition, the silver coating is fairly uniform, with no visible large silver grains. Figure $3(\mathrm{~d})$ shows the diffraction pattern produced when the spirals were illuminated with white light. As it can be seen, the pattern is regular, symmetric, and having welldefined colours, indicating the periodicity of the structures. This periodicity is not disturbed by the support structures.

\section{Conclusions}

To conclude, we have employed DA-DLW and EP of a metalbinding hybrid material to make helical spirals and woodpile structures with $600 \mathrm{~nm}$ intralayer periodicity. The fabricated nanostructures have a smooth surface and exhibit welldefined diffraction spectra, indicating their good fabrication quality and internal periodicity. We have shown that this methodology combination can be employed as a viable route 
for the fabrication of 3D dielectric, nanoparticle-coated, and metallic photonic nanostructures.

\section{Acknowledgments}

This work was partly funded by the ITN TOPBIO (PITNGA-2010-264362). G.Bickauskaite was supported by the EU Marie Curie Fellowship Program: FASTQUAST (PITNGA-2008-214962). A. Pikulin and N. Bityurin were supported in part by Federal Targeted Program "Scientific and scientific-pedagogical personnel of the innovative Russia" under Contract nos. 16.740.11.0656 and 16.740.11.0018, RFBR under Grant nos. 09-02-00665-a and 11-02-97053r_povoljie_a, and by the Program of presidium of the Russian Academy of Sciences "Extreme light fields and applications." It was also supported by Dr. Athanasios Gavrielides from the AFOSR/EORD London Office via the Grant FA8655-11-13090. The authors would like to thank Mrs. Aleka Manousaki for expert technical assistance with SEM.

\section{References}

[1] S. Juodkazis, V. Mizeikis, and H. Misawa, "Three-dimensional microfabrication of materials by femtosecond lasers for photonics applications," Journal of Applied Physics, vol. 106, no. 5, Article ID 051101, 2009.

[2] L. Amato, Y. Gu, N. Bellini et al., "Integrated three-dimensional filter separates nanoscale from microscale elements in a microfluidic chip," Lab on a Chip, vol. 12, no. 6, pp. 11351142, 2012.

[3] M. Malinauskas, A. Zukauskas, K. Belazaras et al., "Laser fabrication of various polymer micro-optical components," The European Physical Journal Applied Physics, vol. 58, Article ID 20501, 8 pages, 2012.

[4] E. Brasselet, M. Malinauskas, A. Žukauskas, and S. Juodkazis, "Photopolymerized microscopic vortex beam generators: precise delivery of optical orbital angular momentum," Applied Physics Letters, vol. 97, no. 21, Article ID 211108, 2010.

[5] T. S. Drakakis, G. Papadakis, K. Sambani et al., "Construction of three-dimensional biomolecule structures employing femtosecond lasers," Applied Physics Letters, vol. 89, no. 14, Article ID 144108, 2006.

[6] V. Melissinaki, A. A. Gill, I. Ortega et al., "Direct laser writing of 3D scaffolds for neural tissue engineering applications," Biofabrication, vol. 3, Article ID 045005, 2011.

[7] S. Engelhardt, E. Hoch, and K. Borchers, "Fabrication of $2 \mathrm{D}$ protein microstructures and 3D polymer-protein hybrid microstructures by two-photon polymerization," Biofabrication, vol. 3, Article ID 3025003, 2011.

[8] V. Mizeikis, S. Juodkazis, R. Tarozaite, J. Juodkazyte, K. Juodkazis, and H. Misawa, "Fabrication and properties of metalodielectric photonic crystal structures for infrared spectral region," Optics Express, vol. 15, no. 13, pp. 8454-8464, 2007.

[9] A. Radke, T. Gissibl, T. Klotzbücher, P. V. Braun, and H. Giessen, "Three-dimensional bichiral plasmonic crystals fabricated by direct laser writing and electroless silver plating," Advanced Materials, vol. 23, no. 27, pp. 3018-3021, 2011.

[10] M. D. Turner, G. E. Schrõder-Turk, and M. Gu, "Fabrication and characterization of three-dimensional biomimetic chiral composites," Optics Express, vol. 19, no. 10, pp. 10001-10008, 2011.
[11] S. W. Hell and J. Wichmann, "Breaking the diffraction resolution limit by stimulated emission: stimulated-emissiondepletion fluorescence microscopy," Optics Letters, vol. 19, no. 11, pp. 780-782, 1994.

[12] T. A. Klar, S. Jakobs, M. Dyba, A. Egner, and S. W. Hell, "Fluorescence microscopy with diffraction resolution barrier broken by stimulated emission," Proceedings of the National Academy of Sciences of the United States of America, vol. 97, no. 15, pp. 8206-8210, 2000.

[13] T. F. Scott, B. A. Kowalski, A. C. Sullivan, C. N. Bowman, and R. R. McLeod, "Two-color single-photon photoinitiation and photoinhibition for subdiffraction photolithography," Science, vol. 324, no. 5929, pp. 913-917, 2009.

[14] N. Li, R. R. Gattass, E. Gershgoren, H. Hwang, and J. T. Fourkas, "Achieving $\lambda / 20$ resolution by one-color initiation and deactivation of polymerization," Science, vol. 324, no. 5929, pp. 910-913, 2009.

[15] J. Fischer and M. Wegener, "Three-dimensional direct laser writing inspired by stimulated-emission-depletion microscopy," Optical Materials Express, vol. 1, pp. 614-624, 2011.

[16] Y. Cao, Z. Gan, and B. Jia, "High-photosensitive resin for super-resolution direct-laser-writing based on photoinhibited polymerization," Optics Express, vol. 19, pp. 19486-19494, 2011.

[17] T. J. A. Wolf, J. Fischer, and M. Wegener, "Pump-probe spectroscopy on photoinitiators for stimulated-emissiondepletion optical lithography," Optics Letters, vol. 36, pp. 3188-3190, 2011.

[18] I. Sakellari, E. Kabouraki, D. Gray et al., "Diffusion-assisted high resolution direct femtosecond laser writing," ACS Nano, vol. 6, no. 3, pp. 2302-2311, 2012.

[19] K. Terzaki, N. Vasilantonakis, A. Gaidukeviciute et al., "3D conducting nanostructures fabricated using direct laser writing," Optical Materials Express, vol. 1, pp. 586-597, 2011.

[20] S. John, "Strong localization of photons in certain disordered dielectric superlattices," Physical Review Letters, vol. 58, no. 23, pp. 2486-2489, 1987.

[21] E. Yablonovitch, "Inhibited spontaneous emission in solidstate physics and electronics," Physical Review Letters, vol. 58, no. 20, pp. 2059-2062, 1987.

[22] K. M. Ho, C. T. Chan, and C. M. Soukoulis, "Existence of a photonic gap in periodic dielectric structures," Physical Review Letters, vol. 65, no. 25, pp. 3152-3155, 1990.

[23] C. M. Soukoulis and M. Wegener, "Past achievements and future challenges in the development of three-dimensional photonic metamaterials," Nature Photonics, vol. 5, no. 9, pp. 523-530, 2011.

[24] J. M. Lourtioz, H. Benisty, V. Berger et al., "Photonic crystals: towards nanoscale photonic devices," Physics Today, vol. 59, no. 8, pp. 54-55, 2006.

[25] F. A. Denis, P. Hanarp, D. S. Sutherland, and Y. F. Dufrêne, "Nanoscale chemical patterns fabricated by using colloidal lithography and self-assembled monolayers," Langmuir, vol. 20, no. 21, pp. 9335-9339, 2004.

[26] C. García-Meca, J. Hurtado, J. Martí, A. Martínez, W. Dickson, and A. V. Zayats, "Low-loss multilayered metamaterial exhibiting a negative index of refraction at visible wavelengths," Physical Review Letters, vol. 106, no. 6, Article ID 067402, 2011.

[27] S. Shukla, X. Vidal, E. P. Furlani et al., "Subwavelength direct laser patterning of conductive gold nanostructures by 
simultaneous photopolymerization and photoreduction," ACS Nano, vol. 5, no. 3, pp. 1947-1957, 2011.

[28] G. J. Leggett, "Direct writing of metal nanostructures: lithographic tools for nanoplasmonics research," ACS Nano, vol. 5, no. 3, pp. 1575-1579, 2011.

[29] G. O. Mallory and J. B. Hajdu, Electroless Plating: Fundamentals and Applications, chapter 1, American Electroplaters and Surface Finishers Society, Orlando, Fla, USA, 1990.

[30] S. Hrapovic, Y. Liu, G. Enright, F. Bensebaa, and J. H. T. Luong, "New strategy for preparing thin gold films on modified glass surfaces by electroless deposition," Langmuir, vol. 19, no. 9, pp. 3958-3965, 2003.

[31] N. Vasilantonakis, K. Terzaki, I. Sakellari et al., "Three-dimensional metallic photonic crystals with optical bandgaps," Advanced Materials, vol. 24, pp. 1101-1105, 2012.

[32] A. V. Kabashin, P. Evans, S. Pastkovsky et al., "Plasmonic nanorod metamaterials for biosensing," Nature Materials, vol. 8, no. 11, pp. 867-871, 2009.

[33] J. K. Gansel, M. Thiel, M. S. Rill et al., "Gold helix photonic metamaterial as broadband circular polarizer," Science, vol. 325, no. 5947, pp. 1513-1515, 2009.

[34] S. Turunen, E. Käpylä, K. Terzaki et al., "Pico- and femtosecond laser-induced crosslinking of protein microstructures: evaluation of processability and bioactivity," Biofabrication, vol. 3, Article ID 045002, 2011.

[35] D. Roundy and J. Joannopoulos, "Photonic crystal structure with square symmetry within each layer and a threedimensional band gap," Applied Physics Letters, vol. 82, no. 22, pp. 3835-3837, 2003.

[36] M. Deubel, Three-dimensional photonic crystals via direct laser writing: fabrication and characterization [Doctoral thesis], Universitat Karlsruhe, 2006.

[37] M. Deubel, M. Wegener, S. Linden, and G. Von Freymann, "Angle-resolved transmission spectroscopy of threedimensional photonic crystals fabricated by direct laser writing," Applied Physics Letters, vol. 87, no. 22, Article ID 221104, pp. 1-3, 2005. 

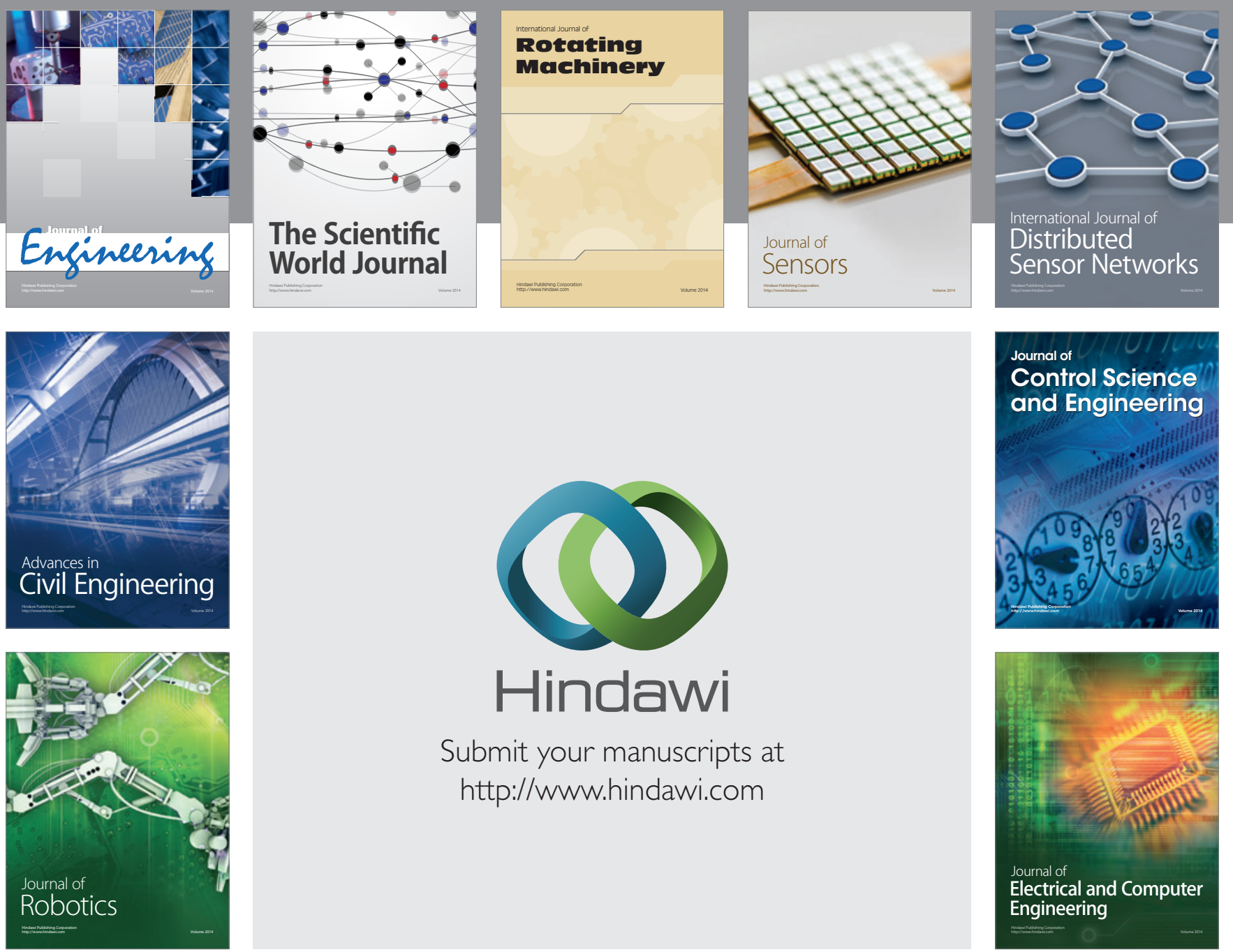

Submit your manuscripts at

http://www.hindawi.com
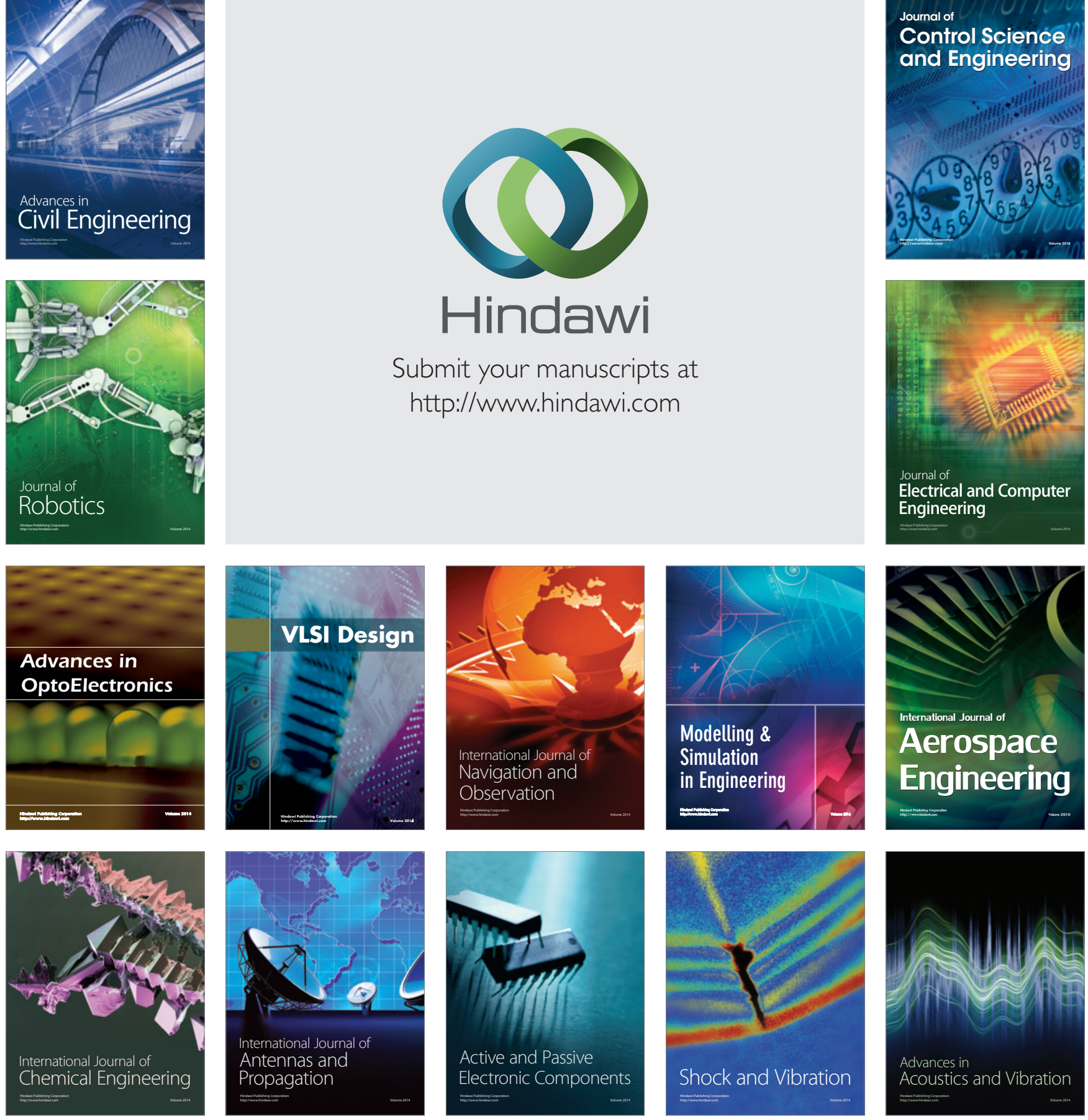